\title{
Maternal-offspring conflict leads to the evolution of dominant zygotic sex determination
}

\author{
JH Werren ${ }^{1}$, MJ Hatcher ${ }^{2}$ and HCJ Godfray ${ }^{3}$ \\ ${ }^{1}$ Department of Biology, University of Rochester, Rochester NY 14627, USA; ${ }^{2}$ School of Biology, University of Leeds, Leeds LS2 \\ 9JT, UK; ${ }^{3}$ NERC Centre for Population Biology, Imperial College at Silwood Park, Ascot, SL5 7PY, UK
}

\begin{abstract}
Sex determination in many species involves interactions among maternally expressed genes (eg, mRNA's and proteins placed into the egg) and zygotically expressed genes. Recent studies have proposed that conflicting selective pressures can occur between maternally and zygotically expressed sex determining loci and that these may play a role in shaping the evolution of sex determining systems. Here we show that such genetic conflict occurs under very general circumstances. Whenever sex ratio among progeny in a family affects the fitness of either progeny in that family or maternal fitness, then maternal-zygotic genetic conflict occurs. Furthermore, we show that this conflict typically results in a 'positive feedback loop' that leads to the evolution of a dominant zygotic sex determining locus. When
\end{abstract}

males more negatively effect fitness within the family, a male heterogametic ( $X Y$ male) sex determining system evolves, whereas when females more negatively effect fitness in the family, a female heterogametic ( $Z W$ female) system evolves. Individuals with the dominant sex allele are one sex, and the opposite sex is determined by maternally-expressed genes in individuals without the dominant sex allele. Results therefore suggest that maternal-zygotic conflict could play a role in the early evolution of chromosomal sex determining systems. Predictions are made concerning the patterns of expression of maternal and zygotic sex determining genes expected to result from conflict over sex determination.

Heredity (2002) 88, 102-111. DOI: 10.1038/sj/hdy/6800015

Keywords: sex determination; parent-offspring conflict; heterogamety

\section{Introduction}

Animals and plants display an extraordinary variety of mechanisms for sex determination (White, 1973; Bull, 1983). This variety is surprising because we might reason that such an important developmental pathway would be highly conserved. However, even within orders and genera, sex determination varies and evolutionary transitions between mechanisms are thought to be frequent. For instance, reptiles exhibit male heterogamety (XY males with heteromorphic sex chromosomes, $X X$ females with homomorphic sex chromosomes), female heterogamety, homomorphy with dominant male or femaledetermining alleles, environmental sex determination, and mixed genetic/environmental systems (Janzen and Paukstis, 1991; Girondot et al, 1994; Viets et al, 1994). Similarly, the Diptera show a diversity of genetic mechanisms, including X:Autosomal balance (eg, Drosophila), dominant male determining loci with heteromorphic or homomorphic sex chromosomes, dominant female determining loci, and maternal sex determination (Ullerich, 1984; Marin and Baker, 1998). The housefly (Musca domestica) shows a variety of sex determining alleles, including maternal effect and zygotically expressed genes (Düebendorfer et al, 1992; Schmidt et al, 1997). Molecular studies indicate that, although certain

Correspondence: JH Werren, Department of Biology, University of Rochester, Rochester NY 14627, USA basal genes involved in sex determination may be conserved, the upstream regulators of sex determination are variable between species (Wilkins, 1995; Sievert et al, 1997; Raymond et al, 1998; Meise et al, 1998; Marin and Baker, 1998).

Why are sex determining mechanisms so diverse? It has long been recognized that conflicting selective pressures over sex determination exist between autosomal genes and non-Mendelian factors (eg, cytoplasmic elements, meiotic driving sex chromosomes; Lewis, 1941; Howard, 1942; Hamilton, 1967). Various authors have proposed that such 'genetic conflict' may cause evolutionary change in sex determination mechanisms (Eberhard, 1980; Cosmides and Tooby, 1981; Werren et al, 1988; Hurst et al, 1996; Werren and Beukeboom, 1998). The basic reasoning behind this idea is that the distortions in sex ratio caused by such non-Mendelian elements creates strong selection for alterations in sex determination that increase production of the rarer sex, because such genotypes have increased fitness. For instance, theoretical models suggest that coevolutionary feedback between cytoplasmic and autosomal factors may lead to the evolution of monogeny (Werren, 1987) and evolutionary transition from female heterogamety to male heterogamety (Rigaud et al, 1997; Caubet et al, 2000). Similarly, sex chromosome meiotic drive has been proposed as a possible mechanism causing the evolution of novel sex determining mechanisms in mammals, such as $X^{*} Y$ females in lemmings (Bull and Bulmer, 1981) and $X Y^{*}$ females in Akodon rodents (Hoekstra and Hoekstra, 2001). 
A more subtle form of sex determination conflict can occur between maternal effect genes (eg, genes producing maternal products such as mRNA or proteins that are placed in the egg and effect zygotic development) and zygotically expressed genes (Werren and Beukeboom, 1998; Werren and Hatcher, 2000). There is growing evidence that maternal effect genes influence sex determination in a wide range of organisms. Maternal effect sex determining genes have been described in Drosophila melanogaster (Steinemann-Zwicky et al, 1990; Cline, 1993), Musca domestica (Schmidt et al, 1997; Düebendorfer and Hediger, 1998), Caenorhabditis elegans (Ahringer et al, 1992) and Chrysomia rufescens (Ullerich, 1984). In many coccids (Nur, 1989), sex of the progeny is determined by the maternal genotype, almost certainly due to maternal effect products placed into the egg. As the genetic details of more systems are discovered, maternal effects on sex determination are likely to be found to be a common feature.

Werren and Beukeboom (1998) suggested a number of scenarios under which maternal effect-zygotic gene conflict might arise over sex determination, including (a) partial inbreeding or local mate competition, and (b) maternal or offspring fitness varying with family sex ratio. The former scenarios were examined by Werren and Hatcher (2000), who found that maternal and zygotic optima for sex determination did indeed differ under partial sib-mating and local mate competition (Hamilton, 1967). As predicted, the maternal optima in both cases were more strongly skewed towards females. Although the zygotic optima were also female-biased, they were less biased than the maternal ESS. Despite rather small differences in the optima, under many circumstances genes producing extreme sex ratios had large initial rates of increase against the alternative strategy, suggesting that slight differences in optima could lead to extremes in sex determining strategies.

Trivers (1974) first realized that maternal and offspring sex ratio optima can diverge, and derived the optimal solutions for the situation where the costs of producing a male and female offspring differ to the parent. However, this result has been largely ignored, and its implications for the evolution of sex determination have not been explored. Here, we consider modifications of Trivers' original approach, and consider their implications for the evolution of sex determining systems. We show that whenever the sex ratio in a family affects the fitness of either the progeny within that family or the maternal fitness, then selection acting upon maternal-effect and zygotic sex determination genes will diverge. Furthermore, we show that coevolution of the maternal-effect and zygotic sex determining genes can lead to the evolution of single zygotic locus sex determination, a likely primitive precursor to male heterogametic $(X Y)$ or female heterogametic (ZW) sex determination (Rice, 1987; Charlesworth, 1996). We also show that such coevolution can, in different circumstances, lead to monogeny (production of all-male or all-female families based on maternal genotype). These models may be relevant to a variety of biological systems. The applicability of the models to real biological systems is discussed.

The basic model

Consider a species in which the sex of an individual is determined either by the genotype of the mother (ie, by maternal effect genes), by the individual's genotype (zygotic effect genes), or by some interaction between these two components. Further, assume that the fitness of the progeny in a family is a function of the family sex ratio (ie, the proportion of males among the progeny of the female parent). For simplicity, we assume that the family sex ratio has an equal effect on the fitness of male or female progeny. As will be seen later, this model can also be used to study situations where family sex ratio influences maternal fitness or family size. To examine the potential for conflicting selective pressures between sexdetermination genes that are expressed in the mother and zygote, we start by comparing the optimal sex ratios for each. We demonstrate that these optima are different and that there is a potential for conflict. We then go on to demonstrate that this conflict can lead to the evolution of diverging sex determining systems, by analysing the joint dynamics of maternal and zygotically expressed sex determination genes.

There are two mathematical strategies for analyzing problems such as these, one employing the machinery of inclusive fitness (eg, Charnov, 1982), the other using more explicit population genetic approaches. The inclusive fitness approach is used to determine evolutionary optima and is versatile in that it can be applied to a relatively diverse set of biological conditions (Taylor, 1994; Taylor and Frank, 1996). However, it assumes weak selection and only small additive genetic deviations from the optimal phenotype. The population genetic approach makes more explicit assumptions about the genetic architecture of a trait, and therefore can be applied to some of the conditions we plan to explore below (eg, invasion of a dominant masculinizing sex determining allele). However, the latter approach is less versatile because very specific assumptions concerning the genetic architecture and population structure are required. We use the inclusive fitness approach to determine the optimal sex ratio for maternally and zygotically expressed genes. Explicit genetic models are then employed to confirm the results of the inclusive fitness approach and to explore the dynamics of major effect alleles. Finally, simulations are used to confirm results from the genetic and inclusive fitness models.

\section{Optimal sex ratios for maternal and zygotic genes}

For both analytical approaches, we consider the dynamics of a rare mutant whose sex ratio strategy deviates from that of the rest of the population. The following terms are defined: $x$ is the mutant sex ratio (proportion males), $\hat{x}$ is the sex ratio of the common genotype and the resident sex ratio strategy, $\omega(\bar{x})$ is the fitness of all members of the family (both males and females), which is a function of the average proportion of males in the family, $\bar{x}$. For much of our analysis, we will use a simple linear function, $\omega(\bar{x})=\alpha+\beta \bar{x}$ where $\alpha$ and $\beta$ are constants. If $\beta>0$, individuals in male-biased broods have higher fitness and other things being equal we expect $a$ male-biased sex ratio to evolve. If $\beta<0$ female-biased broods have higher fitness and a female-biased sex ratio should evolve.

First, the inclusive fitness approach is used to determine the optimum sex ratio for a maternally-expressed gene where offspring fitness is influenced by family sex ratio. Consider a female producing a brood of sex ratio $x$ in a population where the vast majority of others 
produce a sex ratio $\bar{x}$. The mutant's fitness, $W_{m}$ is given by a weighted form of the Shaw-Mohler equation (Charnov, 1982) that takes into account the effect of the mutant's sex ratio on brood fitness,

$$
W_{m}=\frac{\omega(x)}{\omega(\hat{x})}\left(\frac{1-x}{1-\hat{x}}+\frac{x}{\hat{x}}\right) .
$$

The ESS sex ratio is found by solving $\left.\frac{\partial W_{m}}{\partial x}\right|_{x=\hat{x}}$ (eg, Charnov, 1982) and with the linear fitness relationship described above is

$$
x_{m}^{*}=\frac{3 \beta-2 \alpha+\sqrt{4 \alpha^{2}+4 \alpha \beta+9 \beta^{2}}}{8 \beta},
$$

where the asterisk and subscript $m$ denote the maternal optimum.

Now consider a zygotically-expressed factor, and specifically a rare mutant which when expressed in the zygote causes its bearer to become male with probability $x$. If there are $n$ other individuals in the brood then the average brood sex ratio is $\frac{1}{n}(x+(n-1) \hat{x})$. In the inclusive fitness approach we tally the effect of this single mutant's behaviour on its own fitness, and on that of its siblings weighted by their coefficient of relatedness, $r$ (Hamilton, 1964). The mutant's inclusive fitness, $W_{z}$, can be expressed in Shaw-Mohler form

$$
\begin{aligned}
& W_{z}=\frac{\omega\left(\frac{1}{n}(x+(n-1) \hat{x})\right)}{\omega(\hat{x})} \\
& \left(\frac{\frac{1}{n}((1-x)+r(n-1)(1-x))}{(1-\hat{x})}+\frac{\frac{1}{n}(x+r(n-1) x)}{\hat{x}}\right),
\end{aligned}
$$

where the two terms in parentheses are the relative fitness gains through female and male function (both via self and siblings) respectively. The optimum zygotic sex ratio, $x_{z}^{*}$, is found as before,

$$
x_{z}^{*}=\frac{\beta \phi-2 \alpha n+\sqrt{4 \alpha n^{2}(\alpha+\beta)+\beta^{2} \phi^{2}}}{2 \beta(n+\phi)}
$$

where $\phi=n+2(1+(n-1) r)$.

We digress slightly to discuss exactly what we mean by brood size, $n$. In the mutant offspring fitness equation, the unilateral change in sex ratio by the mutant affects the fitness of $n$ other offspring. It does not actually matter whether these other offspring are contemporaneous or are part of the parent's future reproductive success so long as the offspring's sex ratio decision affects its own and their fitness. As optimum offspring sex ratio asymptotes quite fast with increasing $n$ we shall henceforth assume $n \rightarrow \infty$ in which case

$$
x_{z}^{*}=\frac{\beta(1+2 r)-2 \alpha+\sqrt{4 \alpha(\alpha+\beta)+\beta^{2}(1+2 r)^{2}}}{4 \beta(1+r)} .
$$

Note that we re-obtain the parental optimum (Equation 2) if $r=1$ (which implies that the offspring, like the parent, values brood mates identically to itself) and also from equation 4 , if $n=1$ (there are no brood mates for the parent and offspring to treat differently). The optimum sex ratio in the case of full sibs $\left(r=\frac{1}{2}\right)$ is

$$
x_{z}^{*}=\frac{\beta-\alpha+\sqrt{\alpha(\alpha+\beta)+\beta^{2}}}{3 \beta} .
$$

Both equations 2 and 6 are also obtained using an explicitly population genetic approach (see Appendix).

Figure 1 shows the optimal sex ratio for the parent, for the zygote in a family of full siblings, and for the zygote in a family of half siblings. Except when $\beta=0$ (no sex ratio effect on family fitness) the maternal and zygotic optima are different. If individuals in male-biased broods have higher fitness $(\beta>0)$, then more male-biased sex ratios are favoured. However, maternally-expressed genes are selected to produce a more male-biased sex ratio than are zygotically-expressed genes. As relatedness among zygotes in the family decreases (eg, half-sibs; $r=$ $\frac{1}{4}$ ), the zygotic optimum becomes less male biased and potential conflict between maternal and zygotic genes increases. When female-biased families have higher fitness, then the reverse pattern results.

The conflict arises because sex ratio selection is affected by two factors: (i) the Fisherian advantage of producing the rarer sex which tends to result in a sex ratio of equality at equilibrium, and (ii) the family-level advantage of producing a greater proportion of the less damaging (or less costly) sex. Parents and young weight these effects slightly differently, with selection acting on a zygoticallyexpressed gene emphasizing (i) over (ii) relative to a maternally expressed gene, hence leading to a sex ratio nearer equality. There is thus a potential for conflict and this will be greater in species with multiply mating females where sibling relatedness is lower.

\section{Coevolution of maternal sex ratio and a dominant zygotic masculinizer}

The above solutions demonstrate the potential for conflict, but they do not address the evolutionary dynamics of such conflict. We investigate this in the following

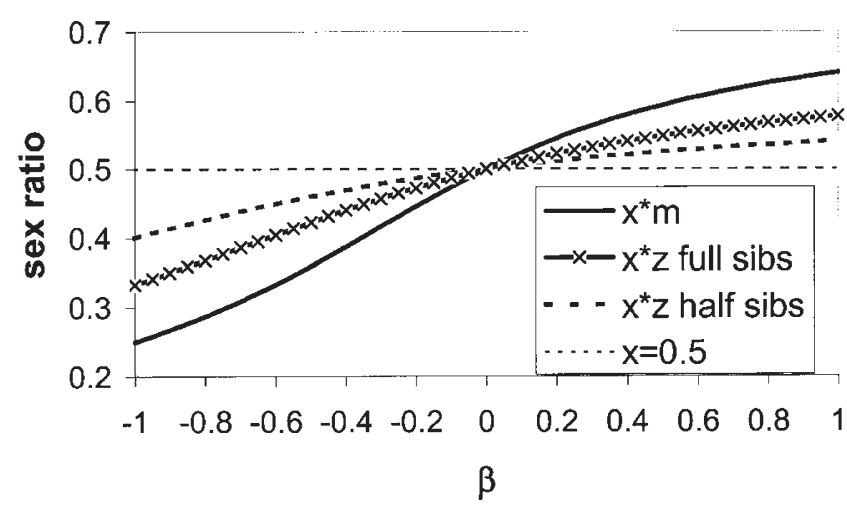

Figure 1 Maternal and zygotic optimal sex ratios in relationship to relative cost of male production $(\beta)$. When $\beta<0$, males reduce brood fitness more than daughters, and a female-biased sex ratio is favoured for both maternal and zygotic genes. Similarly, when daughters are the more detrimental sex $(\beta>0)$, a male-biased sex ratio is selected. Maternally-expressed genes favour a more skewed sex ratio (solid line) than zygotic genes. The zygotic optimum depends on relatedness within broods: genes expressed in full-sibships (crossed line) are selected to bias the sex ratio more than genes in half-sibships (dashed line). 
sections, where the co-dynamics of maternal and zygotic genes are analyzed using a population genetic approach. Specifically, we first investigate the co-dynamics of an allele that causes male development (a dominant masculinizer) at a zygotic sex determination locus, and alleles at a maternal effect locus. We are particularly interested here in whether conflict can lead to an $X X$ female, $X Y$ male sex determination system.

Consider two loci, the first coding for a maternallyexpressed factor that determines brood sex ratio. $a a$ is the common genotype and such females produce a brood sex ratio of $\hat{x}$ (though this may be overridden by a zygotically expressed factor). $A a$ is a mutant genotype where the dominant $A$ allele causes the mother to produce a sex ratio of $x$. The second locus is expressed in the zygote; individuals with the $\mathrm{mm}$ genotype become male with probability $x$ or $\hat{x}$ depending on their mother's genotype, while the mutant $M m$ genotype causes its bearer to become male, regardless of maternal genotype. The $M$ allele is thus a dominant masculinizer. Because $M m$ males can only mate with $\mathrm{mm}$ females the $M M$ genotype never occurs. Let the fraction of males with the $M m$ genotype be $q$.

How will maternal sex ratio, $x$, and the frequency of the zygotically-determined males, $q$, coevolve? We investigate this by first calculating equilibrium $q^{*}$ given $x$, and then the optimal maternal sex ratio $x_{m}^{*}$ given $q$.

Frequency of $\mathrm{Mm}$ males: If the frequency of $\mathrm{Mm}$ among males in the current generation is $q$ then the frequency in the next $\left(q^{\prime}\right)$ will be the number of $M m$ progeny produced divided by the total number of male progeny, taking into account the fitness effect of brood sex ratio on all offspring as before. Thus

$$
q^{\prime}=\frac{q^{\frac{1}{2} \omega}\left(\frac{x+1}{2}\right)}{q \frac{x+1}{2} \omega\left(\frac{x+1}{2}\right)+(1-q) x \omega(x)},
$$

and at equilibrium, $q^{*}$

$$
q^{*}=\frac{\frac{1}{2} \omega\left(\frac{x+1}{2}\right)-x \omega(x)}{\frac{x+1}{2} \omega\left(\frac{x+1}{2}\right)-x \omega(x)}
$$

Optimal maternal sex ratio: We now consider the optimal maternal sex ratio in a population containing $\mathrm{Mm}$ males at frequency $q$ among males. With random mating and assuming $A$ is sufficiently rare such that $A A$ genotypes and $A a \times A a$ matings can be ignored, the transmission dynamics of the $A$ allele in a population of $a a$ individuals can be represented in matrix format by $\epsilon_{i}^{\prime}=\mathbf{G} \epsilon_{i}$, or

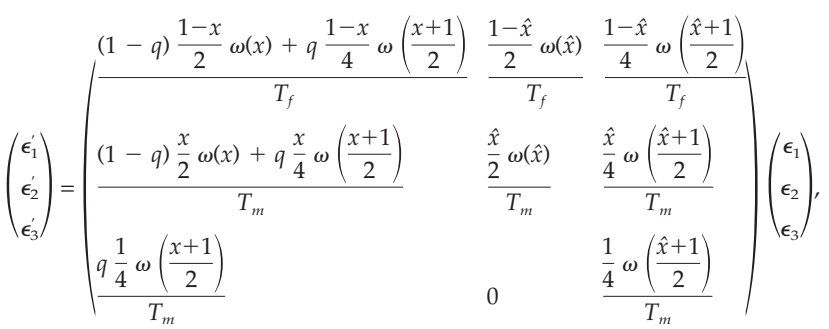

where $\epsilon_{i}$ is the sex-specific frequency of one of the three genotype/sex combinations containing the $A$ allele (Aamm females, $i=1$; Aamm males, $i=2$; AaMm males, $i=3$ ). To obtain relative gene frequencies we divide by $T_{f}$ or $T_{m}$, the total number of females and males produced that generation

$$
\begin{aligned}
T_{f} & =(1-q)(1-\hat{x}) \omega(\hat{x})+q \frac{1-\hat{x}}{2} \omega\left(\frac{\hat{x}+1}{2}\right) \\
T_{m} & =(1-q) \hat{x} \omega(\hat{x})+q \frac{\hat{x}+1}{2} \omega\left(\frac{\hat{x}+1}{2}\right)
\end{aligned}
$$

Whether the rare allele spreads depends on the value of the dominant eigenvalue $(\lambda)$ of the matrix $\mathbf{G}$. The optimal maternal sex ratio $x_{m}^{*}$ is found by calculating $\left.\frac{\partial \lambda}{\partial x}\right|_{x=\hat{x}}=0$ and checking that $\lambda$ is at a maximum rather than a minimum (eg, Charnov, 1982).

Analytical solution of the maternal optimum is possible if the function $\omega$ is specified but even with the simplest linear form the result is too complicated to be informative. We thus proceed by numerical solutions, though note two special cases. First, if $\omega$ is a constant (ie, no effect of brood composition on fitness) then $x_{m}^{*}=$ $(1-q) /(2-q)$ and the overall population sex ratio is $\frac{1}{2}$. As has long been appreciated, the Fisherian sex ratio can be achieved by all females producing half sons and half daughters, or half the females producing sons and the other half daughters, or, as here, a combination of the two. Second, if $M m$ males are absent $(q=0)$ then the pure effect of brood composition on sex ratio can be studied, for example if $\omega=\alpha+\beta x$ then $x_{m}^{*}=\frac{1}{8 \beta}(3 \beta-2 \alpha+$ $\sqrt{4 \alpha^{2}+4 \alpha \beta+9 \beta^{2}}$ ) which is exactly the same result as that derived earlier for the maternal optimum using the inclusive fitness approach (Equation 2).

Co-dynamics of $M m$ and maternal sex ratio: Given the frequency of $M m$ males as a function of maternal sex ratio, and the optimum sex ratio of maternally-expressed genes to different frequencies of $\mathrm{Mm}$, the co-dynamics of the system can be explored. The dynamics of the system are quite interesting. Basically, when males have a negative effect on family fitness $(\beta<0)$, a positive feedback between the maternal effect sex ratio and the frequency of $\mathrm{Mm}$ occurs. This positive feedback results in the evolution of dominant male zygotic sex determination. Figure $2 a$ illustrates the case where males have a mild negative effect on family fitness $(\beta=-0.1)$. In the absence of $\mathrm{Mm}$ males, this leads to a slightly female-biased maternal sex ratio (in this particular case $x_{m}^{*}=0.474$ ). However, this female-biased sex ratio allows the $M$ allele to invade and to equilibrate at a frequency of 0.051 . $M$ spreads for the reasons described in the first section: it is a zygoticallyexpressed gene and compared with maternally-expressed genes it experiences stronger Fisherian frequency-dependent selection, and weaker selection to produce less of the sex that reduces family-level fitness. Given that the frequency of $\mathrm{Mm}$ males is 0.051 a lower maternal sex ratio is selected (0.461) and this leads to a greater value of $q$ and so on. As is plotted in Figure 2a, the system evolves until $q=1$ and all males are $M m$ and $x_{m}^{*}=0$ and all females are $m m$. This shows that the system can evolve to a dominant male zygotic sex determination ( $\mathrm{Mm}$ male, 
a

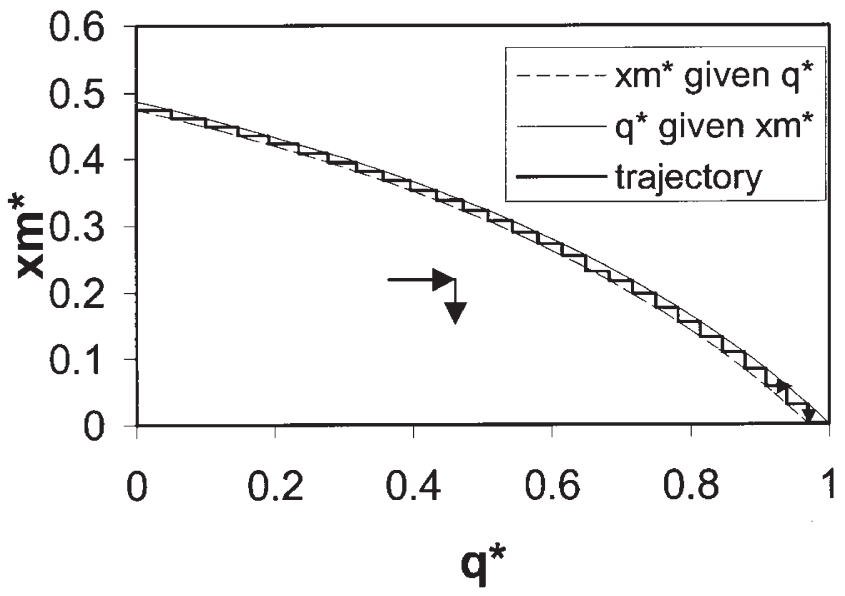

b

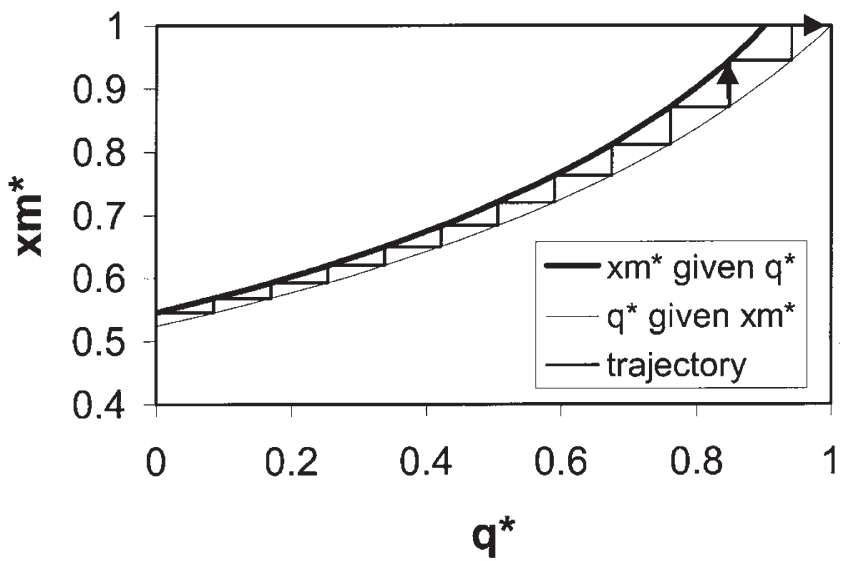

Figure 2 (a) Maternal effect optima and proportion $M m$ zygotes among males when males reduce brood fitness more than females $(\beta=-0.1)$. A positive feedback between the dominant male allele and maternal feminizing alleles leads to male heterogamety $(\mathrm{Mm}$ males and $m m$ females). The population starts at the maternal sex ratio optimum and $0 \% \mathrm{Mm}$ males. It evolves along the line of evolutionary trajectory indicated by the arrows, by a 'positive feedback' between maternal and zygotic sex determining loci, reaching the end-point where $100 \%$ of males are $\mathrm{Mm}$ and the maternal locus produces $0 \%$ males ( $\mathrm{mm}$ females). (b) Maternal effect optima and proportion $\mathrm{Ff}$ zygotes among females when females reduce brood fitness more than males $(\beta=0.2)$. When females are the more 'costly' sex, the system evolves to female heterogamety ( $F f$ females and ff males; arrows as above).

$\mathrm{mm}$ female) as a result of the maternal-zygotic genetic conflict. Such dominant single locus male sex determination occurs in many organisms, including a number of systems with cytologically heteromorphic sex chromosomes (XY male XX females), where a dominant male determiner occurs on the $\mathrm{Y}$. Degenerate $\mathrm{Y}$ chromosomes are believed to evolve from dominant single locus sex determination by a number of mechanisms (Charlesworth, 1978; Rice, 1994). Such a system can be considered analogous to a primitive (cytologically undifferentiated) sex chromosome system with male heterogamety, where a dominant male determiner occurs on the analogue to the Y chromosome (Bull, 1983). Such dominant single locus male sex determination occurs in many organisms, including systems with $(X Y$ male $X X$ females) but without cytologically heteromorphic sex

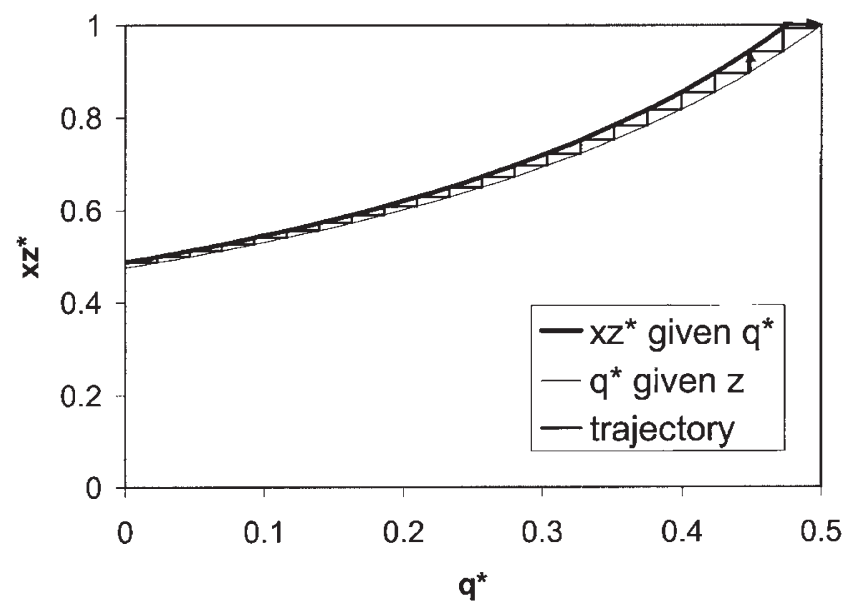

Figure 3 Evolution to monogeny: a maternal feminizer overrides a zygotic male determining locus $(\beta=-0.1)$. Complete monogeny evolves, with the population being composed of $F f$ mothers that produce all daughters and $f f$ mothers that produce all sons $\left(x_{z}^{*}=1\right)$. As above, the population sex ratio thus evolves to 50:50 $\left(q^{*}=0.5\right)$. Arrows indicate the trajectory of evolutionary change.

chromosomes. The evolution of heteromorphic sex chromosomes from undifferentiated precursors has been demonstrated theoretically and results from selection for reduced recombination, which can occur for a variety of reasons (Charlesworth, 1978, 1996; Charlesworth and Charlesworth, 1980; Rice, 1987, 1994).

An interesting and counter-intuitive outcome of maternal-effect zygotic conflict is that the population sex ratio evolves to $50 \%$ males. This occurs even though the 'optimal' sex ratio for both maternal effect and zygotic genes is female-biased (Figure 1). The reason for this is that the presence of the conflict over sex ratio allows the successive invasion of increasingly biased maternallyand zygotically-expressed factors that ultimately reach a hard constraint (the frequency of $\mathrm{Mm}$ males cannot exceed one, and maternal sex ratio cannot be smaller than zero) that results in the equal sex ratio. We have explored the co-dynamics of the two loci for different values of $\beta$ $<0$ and for different forms of the function $\omega$ and believe our results are robust whenever there is conflict of this type with males having a greater negative effect on family fitness than females. The analysis presented here relies on invasion analysis for the maternal allele, $A$, but we have also simulated the complete dynamics of the system to check that invasion by $A$ implies it achieves fixation.

The analysis above assumes that the maternal effect locus evolves incrementally by replacement of alleles of small effect relative to resident alleles, in response to the dominant zygotic masculinizing allele. Is the positive feedback dependent upon small incremental changes at the maternal effect locus? We investigated this by looking at the dynamics of a major maternal effect locus with a dominant masculinizer. In this situation $F_{m} f_{m}$ females produce all-females among the $m m$ progeny ( $\mathrm{Mm}$ progeny develop into males) and $f_{m} f_{m}$ females produce $x=1 / 2$ sons among $\mathrm{mm}$ progeny ( $\mathrm{Mm}$ develop into males). Here, the all-female producing $F_{m} f_{m}$ genotype invades the population whenever $\beta>0$, and positive feedback evolves the system to dominant zygotic masculinizer as before $\left(F_{m} F_{m} M m\right.$ males and $F_{m} F_{m} m m$ females). Therefore, the result is not dependent upon incremental changes at 
the maternal effect locus. The same conclusions result for evolution of ZW females when $\beta>0$ (see below).

\section{Evolution of incipient female heterogamety and monogeny}

Here it is shown that the same type of positive feedback between maternally- and zygotically-expressed sex determination loci in the presence of conflict over the sex ratio can give rise to incipient female heterogamety (primitive ZW female/ZZ male systems) and monogeny (females that produce $100 \%$ males or $100 \%$ females), depending upon the circumstances.

Evolution of female heterogametic sex determination (primitive ZW females): The analysis of the evolution of incipient female heterogamety (again, to the primitive stage with sex chromosomes undifferentiated except at a single sex determining locus) uses the same basic approach as above. Female heterogamety evolves when female progeny are more costly to family fitness $(\beta>0)$. Here we consider a zygotic locus which when ff causes its bearer to become male with a probability, $x$ or $\hat{x}$, dictated by maternal genotype, but which when $F f$ always develops as a female (a dominant feminizer). As Ff individuals are always females they inevitably mate with $f f$ males and no FF genotypes are ever produced. Proceeding exactly as before we can derive the equilibrium frequency of $F f$ as a function of maternal sex ratio, and then derive the optimum maternal sex ratio as a function of the $F f$ frequency. It is easy to show that the dominant feminizer will not invade when $\beta<0$ (ie, when males are the costlier sex to family fitness). However, it does readily invade when $\beta>0$, as described below.

In Figure $2 b$ we illustrate the co-dynamics in the same way as in the analysis of the dominant masculinizer, except that now we assume that females have a slightly greater negative effect on offspring fitness $(\beta=0.2)$. This effect results in a slightly male-biased maternal sex ratio, which allows $F f$ to spread and equilibriate at a low frequency. This initiates the same type of positive feedback observed before, except that now it results in $100 \%$ of females being $\mathrm{Ff}$ and $100 \%$ of males being ff (because the maternal effect locus evolves to $100 \%$ male production, $x_{m}^{*}=1$ ). We thus have incipient female heterogamety, potentially a precursor to the ZW female/ZZ male systems found in birds, some reptiles and fish and invertebrates (White, 1973; Bull, 1983). Note that the positive feedback does not require small incremental changes at the maternal locus; simulations indicate that female heterogamety will also evolve from a positive feedback between a dominant maternal allele that produces $100 \%$ males and a dominant zygotic feminizer (that overrides the action of the maternal locus). As before, despite both optima being male biased, the end result of coevolutionary feedback is a 1:1 population sex ratio.

Evolution of monogeny: In some sex determination systems, sex ratio is apparently determined completely by the genotype of the mother. In these systems, some mothers produce $100 \%$ males whereas others produce $100 \%$ females (White, 1973; Bull, 1983). These are presumably cases where sex is controlled by maternal effect genes. Examples include the fly Chrysomya rufifacies (Ullerich, 1984), some coccid insects (Nur, 1989), midges and cynipid wasps (White, 1973).

A more complete treatment of the dynamics leading to monogeny will be presented elsewhere, and here we present only the basic results. When males negatively affect family fitness, maternal-effect loci are selected to produce a female-biased sex ratio. Above we have shown how this can lead to $X Y$-type sex determination when there is a zygotic, dominant masculinizer that overrides the action of a maternal sex determining locus. However, now consider a maternally-expressed allele $\left(F_{m}\right)$ that causes its bearer $\left(F_{m} f_{m}\right)$ to produce all-female broods, and that overrides the action of a zygotic locus; the progeny of $f_{m} f_{m}$ mothers develop into the sex based upon their genotype at the zygotic locus ( $a a$ or $A a$ ), whereas $F_{m} f_{m}$ mothers produce all-female progeny. Following the same approaches as before, the equilibrium frequency $\left(q^{*}\right)$ of $F_{m} f_{m}$ females is $q^{*}=\frac{\frac{1}{2} \omega(0)-(1-x) \omega(x)}{\omega(0)-(1-x) \omega(x)}$, and the zygotic optimum in the presence of such females can be found from analysis of the matrix:

$$
\left(\begin{array}{c}
\epsilon_{1}^{\prime} \\
\epsilon_{21}^{\prime} \\
\epsilon_{31}^{\prime}
\end{array}\right)=\left(\begin{array}{ccc}
\frac{(1-x) \omega\left(\frac{x+\hat{x}}{2}\right)}{2 T_{f}} \frac{\frac{1}{4} q \omega(0)+\frac{1}{2}(1-q)(1-x) \omega\left(\frac{x+\hat{x}}{2}\right)}{T_{f}} & \frac{\omega(0)}{4 T_{f}} \\
\frac{x \omega\left(\frac{x+\hat{x}}{2}\right)}{2 T_{m}} & \frac{(1-q) x \omega\left(\frac{x+\hat{x}}{2}\right)}{2 T_{m}} & 0 \\
0 & \frac{q \omega(0)}{4 T_{f}} & \frac{\omega(0)}{4 T_{f}}
\end{array}\right)\left(\begin{array}{c}
\epsilon_{1} \\
\epsilon_{2} \\
\epsilon_{31}
\end{array}\right)
$$

with: $T_{m}=(1-q) \hat{x} \omega(\hat{x}), T_{f}=q \omega(0)+(1-q)(1-\hat{x}) \omega(\hat{x})$, and $\epsilon_{i}$ the frequencies of $A a f_{m} f_{m}$ females $(i=1), A a f_{m} f_{m}$ males $(i=2)$ and $A a F_{m} f_{m}$ females $(i=3)$.

We can show that under some circumstances, the $F_{m} f_{m}$ genotype will spread through the population to a frequency of 0.5 , while the zygotic sex locus optimum declines to $0 \%$ males ( $x_{z}^{*}=0$; Fig. 3 ). The result is monogeny; a population of females producing either $100 \%$ daughters $\left(F_{m} f_{m}\right)$ or $0 \%$ daughters $\left(f_{m} f_{m}\right)$. Our analysis of the co-dynamics indicates that monogeny will not necessarily evolve; for $\beta<-0.5$ an internal equilibrium exists such that the frequency of $F_{m} f_{m}<0.5$ and $x_{z}^{*}<1$. Similarly, when females are more costly to family fitness ( $\beta$ $>0$ ), then a dominant all-male maternal effect allele spreads and zygotic alleles evolve towards more female production.

Given that family sex ratio effects on family fitness can evolve either dominant zygotic sex determination ( $X Y$ males and $Z W$ females) or dominant maternal effect alleles and monogeny, the question is which should occur? Based on these models, we conclude that this depends upon whether maternal effect or zygotic genes are 'epistatically' dominant. That is, if a zygotic gene can override the maternal effect contribution, then $X Y$ or $Z W$ systems result. If a maternal effect locus overrides zygotic sex loci, then monogeny can occur. Our analysis indicates that conditions for the evolution of monogeny may be more stringent than those for dominant zygotic sex determination. However, we suspect that other selective effects (eg, inbreeding depression) may also predispose systems to monogeny versus zygotic sex determination (Bull, 1983). 


\section{Discussion}

Trivers (1974) first pointed out the potential for genetic conflict between parents and offspring over reproduction. It is widely known that he considered conflict over resource allocation to progeny (parental investment), and concluded that progeny are generally selected to seek more resources from a parent than the parent is selected to provide, assuming that providing the extra resource imposes a future reproductive cost to the parent. He also extended this thinking to sex ratio evolution (Trivers, 1974) showing that the optimum sex ratio differs for the offspring versus the mother when one sex costs more to produce than does the other. However, the implications of this result to the evolution of sex determination have not been widely considered.

Here we show that even relatively slight effects of family sex ratio on offspring fitness results in genetic conflict over sex determination, between maternally expressed and zygotically expressed genes. This conflict, via a 'positive feedback' between zygotic and maternal effect genes, can lead to the evolution of dominant single locus zygotic sex determination. When male progeny reduce family fitness, a dominant $M m$ male $\mathrm{mm}$ female system evolves; when female progeny reduce family fitness, a dominant Ff female ff male system evolves. Although the outcome can be zygotic 'control' over sex determination, the underlying structure of sex determination should still reveal the conflict. In $\mathrm{Mm}$ male systems, maternally expressed genes are predicted to push sex determination towards female development, and it is for this reason that, in the absence of the dominant $M$ male determiner, the default sex of $\mathrm{mm}$ individuals is female. Similarly, in Ff female systems, maternally expressed genes are predicted to push sex determination towards males, and the default sex in the absence of $F$ (due to the action of maternal effect genes) is male. In both cases, a dominant single sex locus sex determination is expected to evolve when the sex locus can override the influences of maternally expressed genes favoring the opposite sex. A key point here is that even though the sex ratio in the population may be 50:50, with a dominant zygotic sex determining locus, the situation can arise from genetic conflict between zygotic and maternal sex determining factors and the signature of this conflict will be apparent in the expression patterns of zygotic and maternal sex determining genes.

The structure of our model is general enough to be applicable to three different situations: (a) sex ratio in a family affects the fitness (eg, size, survival) of offspring in the family, (b) male and female offspring 'cost' different amounts of resources for the mother to produce, and therefore her family size is influenced by her family sex ratio (in this case the fitness term is family size, which is a function of the sex ratio), and (c) the sex ratio in the family affects longevity or future fecundity of the mother, and therefore her lifetime fitness. How likely are these conditions to exist in nature? Most relevant data come from mammals and birds. Males of many polygynous mammals are thought to be more costly than females, although appropriate cost measurements are rare (Clutton-Brock, 1982; Clutton-Brock and Iason, 1986). Mothers allocate more resources to sons than daughters in red deer, and are less likely to calf having reared a son in the previous winter (Clutton-Brock et al, 1981; Gomen- dio et al, 1990). Gomendio et al (1990) discuss examples where son suckling frequency is higher than that of daughters in red deer, goats, bison and African elephants. There is evidence for sex-biased resource allocation in primates (reviewed in van Hoof (1997)) and some human populations (Bereczkei and Dunbar, 1997). Examples and scenarios for biased provisioning in birds are reviewed in Stamps (1990) and Sheldon (1998). In several bird species, there is now substantial evidence that fledging sex ratios may be determined by maternal-offspring conflict over sex ratio and resource allocation (Dhondt and Hochachka, 2001). In the great tit, Parus major, male chicks are stronger competitors than their female siblings, resulting in more male-biased fledging sex ratios particularly when resources are scarce, the opposite pattern to that favoured by maternal sex ratio genes (Oddie, 2000). Many potential examples of sex ratio dependent maternal fitness may occur when offspring fitness is sex-differentially dependent on maternal condition (Trivers and Willard, 1973) as this model implicitly assumes sex-differential costs of rearing (average) offspring. These circumstances are also likely to be common in insects and do not require extensive parental care to occur. For example, Nunney (1983) found that the size and fecundity of male and female Drosophila are differentially affected by food availability, suggesting that family sex ratio could influence offspring fitness under some circumstances. Another key point is that the fitness effects can be very slight, and still catalyze the evolution of maternally expressed genes that favor one sex and a dominant sex determining allele that favors the opposite sex. For this reason, the conditions favoring the positive feedback that leads to dominant zygotic sex determination are likely to be widespread.

Dominant zygotic sex determination is likely to be a precursor to the evolution of heteromorphic sex chromosomes in some systems; $M m$ male heterogamety leading to the evolution of $X Y$ males with heteromorphic sex chromosomes and a degenerate $\mathrm{Y}$ chromosome, and $\mathrm{Ff}$ female heterogamety leading to the evolution of $\mathrm{ZW}$ females with heteromorphic sex chromosomes and degenerate $\mathrm{W}$ chromosomes. The processes involved in sex chromosome evolution have been examined extensively and are thought to be quite complex (Charlesworth and Charlesworth, 1980; Bull, 1983; Rice, 1987, 1994; Charlesworth, 1996). Once an incipient sex chromosome system (eg, single locus sex determination) has evolved, selection can favor reduced recombination between sex chromosomes for a variety of reasons (Rice, 1987; Charlesworth, 1996). As a result, further heterogeneity characteristic of advanced sex chromosome systems can evolve, including loss of gene function and eventual degeneration of the Y. Less work has concentrated on the processes involved in the initial evolution of dominant single locus sex determining systems.

$X Y$ male/ XX female sex determination occurs in mammals and is common in vertebrates and some insect groups. ZW female/ZZ male systems occur in birds, snakes, lepidopterans (butterflies and moths), some fish and isopods, and other organisms (Bull, 1983). It is not uncommon in the literature for dominant $\mathrm{Mm}$ male sex determination to be described as XY male sex determination (and dominant $F f$ female sex determination as $\mathrm{ZW}$ female sex determination), even in systems without heteromorphic sex chromosomes. Further, many systems with heteromorphic sex chromosomes may involve a 
dominant zygotically-acting sex locus on the heteromorphic sex chromosome.

In mammals, sex determination is likely to have arisen originally from dominant male sex determination, with the dominant sex locus SrY now occurring on the otherwise largely degenerate $Y$ chromosome (Goodfellow and Lovell-Badge, 1993). In Diptera, sex determination ranges from single locus dominant male ( $\mathrm{Mm}$ males), to XY systems containing a dominant sex locus, to $\mathrm{X}$ :Autosomal balance, to monogeny (dominant maternal sex determination) (Marin and Baker, 1998). In systems with $\mathrm{X}$ :Autosomal balance sex determination, such as Drosophila melanogaster, it is less obvious whether dominant single locus sex determination was the ancestral state. However, given the abundance of dominant sex locus sex determination in other dipterans (Marin and Baker, 1998), it is possible that $\mathrm{X}$ :autosome balance systems were also derived ancestrally from dominant $\mathrm{Mm}$ male sex determination. One explanation for the evolution of $\mathrm{X}$ :Autosomal balance is selection for coupling of dosage compensation with sex determination. Dosage compensation is a problem which arises once one sex chromosome has undergone degeneration or gene silencing, such that the heterogametic sex has an unbalanced number of transcriptionally active genes on the sex chromosome. There are a number of theories proposed to explain the evolution of Y (or W) degeneration (Charlesworth, 1978; Rice, 1994).

The results presented here show that likely precursors of $\mathrm{XY}: \mathrm{XX}$ and $\mathrm{ZW:ZZ} \mathrm{heteromorphic} \mathrm{sex} \mathrm{chromosome}$ systems, dominant $M m$ male and dominant $F f$ female sex determination, readily evolve as a consequence of maternal-zygotic genetic conflict over sex determination. To our knowledge, this is one of the few formal models accounting for the evolution of dominant single locus sex determination. Charlesworth and Charlesworth (1978) have investigated analytically the evolution of twozygotic locus systems (involving separate loci for male and female function) for the evolution of separate sexes from a hermaphroditic plant progenitor. Bull (1983: pp 135-137) and Kraak and DeLooze (1993) have suggested other routes to the evolution of single locus sex determination; their theories invoke fluctuations in the population sex ratio for major genes to become established.

A second possible outcome of maternal zygotic gene conflict is the evolution of maternal control over zygotic sex determination. When a dominant maternal locus exists, this will lead to monogeny, where some females produce all-female families and others produce all-male families. Our results show that monogeny can evolve when the maternal locus overrides the action of zygotic sex loci, and when the fitness of the family is affected by sex ratio in the family. Monogeny occurs in a number of dipterans, including some midges and sciarid and calliphorid flies (Ullerich, 1984; Gerbi, 1986; Rocha and Perondini, 2000). It is unclear to us what circumstances will lead to a maternal locus being able to override the action of a zygotic locus, versus the reverse. It would seem that the zygote would have ultimate control over its own sex, and perhaps this explains the much more common occurrence of dominant zygotic sex determination relative to dominant maternal control. However, it is possible that maternal imprinting or silencing of zygotic sex determining loci may provide maternal control in some circumstances.
Both theoretical and empirical studies present growing evidence that genetic conflict can play a role in sex determination evolution (Bull and Charnov, 1977; Juchault and Rigaud, 1995; Carvalho et al, 1998; Werren and Beukeboom, 1998; Caubet et al, 2000; Werren and Hatcher, 2000). The existence of divergent selection between nuclear genes and inherited cytoplasmic factors (eg, mitochondria, chloroplasts, inherited microorganisms) is well established and can lead to polymorphisms in sex determination within populations, such as CMS in plants (Koelewijn and van Damme, 1995; Frank, 2000) and cytoplasmic sex ratio distorters in animals (Juchault et al, 1993; Kelly et al, 2001). Conflict between driving sex chromosomes and autosomal genes may similarly shape sex determining systems (Hamilton, 1967; Carvalho et al, 1998; Jaenike, 1999).

Added to these more obvious sources of conflicting selective pressures over sex determination, is maternalzygotic gene conflict. Such conflict can result under partial inbreeding (Werren and Hatcher, 2000) or as shown here when fitness of offspring correlates with family sex ratio. The potential consequences of maternal-zygotic sex determination conflict have not been fully explored. For example, more explicit models are needed to investigate the effects of multiple mating, maternal fitness effects, and differential fitness costs to male and female progeny on maternal and zygotic conflict. Also unexplored is potential maternal-paternal conflict over zygotic sex determination. Such conflict could be mediated by paternal and maternal imprinting of sex determining loci; such as proposed for genes involved in offspring accrual of resources during pregnancy (Haig, 1993). As a general pattern, we expect that paternally expressed sex determining genes will be selected to push sex determination more towards a balanced sex ratio (as is the case for zygotic sex determining genes); differential selection on paternal versus maternal sex determiners will increase with multiple mating by the female, either within a single brood or over successive broods, because the 'genetic interests' of maternal and paternal genes diverge more greatly under these circumstances.

We hope that researchers investigating the mechanics of sex determination will consider the possible role of conflicting selective pressures between different genetic elements, particularly between zygotic and maternal sex determining loci, in shaping the underlying structure of sex determination systems.

\section{Acknowledgements}

The work of JHW was supported by funds from the US NSF. MJH was supported by the Royal Society (Dorothy Hodgkin Research Fellowship 502008) and NERC (GR3/11880). We would like to thank Deborah Charlesworth, Brian Charlesworth, and Leo Beukeboom for helpful discussions on sex determination.

\section{References}

Ahringer J, Rosenquist TA, Lawson DN, Kimble J (1992). The Caenorhabditis elegans sex determining gene fem-3 is regulated posttranscriptionally. EMBO 11: 2303-2310.

Bereczkei T, Dunbar RIM (1997). Female-biased reproductive strategies in a Hungarian Gypsy population. Proc $R$ Soc Lond B 264: 17-22. 
Bull JJ, Bulmer MG (1981). The evolution of XY females in mammals. Heredity 47: 347-365.

Bull JJ (1983). The Evolution of Sex Determining Mechanisms. Benjamin/Cummings: Menlo Park, CA.

Bull JJ, Charnov EL (1977). Changes in the heterogametic mechanism of sex determination. Heredity 39: 1-14.

Carvalho AB, Sampaio MC, Varandas FR, Kaczko LB (1998). An experimental demonstration of Fisher's principle: evolution of sexual proportion by natural selection. Genetics 148: 719-731.

Caubet Y, Hatcher MJ, Mocquard J-P, Rigaud T (2000). Genetic conflict and changes in heterogametic mechanisms of sex determination. I Evol Biol 13: 766-777.

Charlesworth B (1978). Model for evolution of Y chromosomes and dosage compensation. Proc Natl Acad Sci USA 75: 56185622.

Charlesworth B (1996). The evolution of chromosomal sex determination and dosage compensation. Curr Biol 6: 149-162.

Charlesworth B, Charlesworth D (1978). A model for the evolution of dioecy and gynodioecy. Am Nat 112: 975-997.

Charlesworth D, Charlesworth B (1980). Sex differences in fitness and selection for centric fusions between sex-chromosomes and autosomes. Gent Res 35: 205-214.

Charnov EL (1982). The Theory of Sex Allocation. Princeton University Press: Princeton, NJ.

Cline TW (1993). The Drosophila sex determination signal: how do flies count to two? Trends Genet 9: 385-390.

Cosmides ML, Tooby J (1982). Cytoplasmic inheritance and intragenomic conflict. J Theor Biol 89: 83-129.

Clutton-Brock TH (1982). Sons and daughters. Nature 298: 11-13.

Clutton-Brock TH, Albon SD, Guinness FE (1981). Parental investment in male and female offspring in polygynous mammals. Nature 289: 487-489.

Clutton-Brock TH, Iason GR (1986). Sex ratio variation in mammals. Q Rev Biol 61: 339-374.

Dhondt A, Hochachka WM (2001). Adaptive sex ratios and parent-offspring conflict. Trends Ecol Evol 16: 61-62.

Dübendorfer A, Hediger M (1998). The female-determining gene $F$ of the housefly, Musca domestica, acts maternally to regulate its own zygotic activity. Genetics 150: 221-226.

Dübendorfer A, Hilfiker-Kleiner D, Nothinger R (1992). Sex determination mechanisms in dipteran insects: the case of Musca domestica. Develop Biol 3: 349-356.

Eberhard WG (1980). Evolutionary consequences of intracellular organelle competition. Q Rev Biol 55: 231-249.

Frank SA (2000). Polymorphism of attack and defencse. Trends Ecol Evol 15: 167-171.

Gerbi SA (1986). Unusual chromosome movements in sciarid flies. In: Hennig W (ed) Results and Problems in Cell Differentiation, vol. 13: Germ Line - Soma Differentation. SpringerVerlag, pp 71-104.

Girondot M, Zaborski P, Servan J, Pieau C (1994). Genetic contribution to sex determination in turtles with environmental sex determination. Genet Res 63: 117-127.

Gomendio M, Clutton-Brock TH, Albon SD, Guinness FE, Simpson MJ (1990). Mammalian sex ratios and variation in costs of rearing sons and daughters. Nature 343: 261-263.

Goodfellow PN, Lovell-Badge R (1993). SRY and sex determination in mammals. Annu Rev Genet 27: 71-92.

Haig D (1993). Genetic conflict in human pregnancy. Q Rev Biol 68: 495-532.

Hamilton WD (1964). The genetical evolution of social behaviour. J Theor Biol 7: 1-52.

Hamilton WD (1967). Extraordinary sex ratios. Science 156: 477-488.

Hoekstra HE, Hoekstra JM (2001). An unusual sex-determination system in South American field mice (genus Akodon): The role of mutation, selection, and meiotic drive in maintaining XY females. Evolution 55: 190-197.

Howard HW (1942). The genetics of Armadillidium vulgare Latrielle. II. Studies on the inheritance and monogeny and amphogeny. Genetics 44: 143-159.
Hurst LD, Atlan A, Bengtssom BO (1996). Genetic conflicts. Q Rev Biol 71: 317-364.

Jaenike J (1999). Suppression of sex ratio meiotic drive and the maintenance of Y-chromosome polymorphism in Drosophila. Evolution 53: 164-174.

Janzen FJ, Paukstis GL (1991). Environmental sex determination in reptiles: ecology, evolution and experimental design. $Q$ Rev Biol 66: 149-179.

Juchault P, Rigaud T, Mocquard JP (1993). Evolution of sex determination and sex ratio variability in wild populations of Armadillidium vulgare (Latr.) (Crustacea, Isopoda): a case study in conflict resolution. Acta Oecologia 14: 547-562.

Juchault P, Rigaud T (1995). Evidence for female heterogamety in two terrestrial crustaceans and the problem of sex chromosome evolution in isopods. Heredity 75: 466-471.

Kelly A, Dunn AM, Hatcher MJ (2001). Population dynamics of a vertically transmitted, parasitic sex ratio distorter and its amphipod host. Oikos 94: 392-402.

Koelewijn HP, van Damme JMM (1995). Genetics of male sterility in gynodioecious Plantago coronopus. II. Nuclear genetic variation. Genetics 139: 1759-1775.

Kraak SBM, De Looze EMA (1993). A new hypothesis on the evolution of sex detemination in vertebrates: big females $\mathrm{ZW}$, big males XY. Netherlands J Zool 43: 260-273.

Lewis D (1941). Male sterility in natural populations of hermaphrodite plants. N Phytol 40: 50-63.

Marin I, Baker BS (1998). The evolutionary dynamics of sex determination. Science 281: 1990-1994.

Meise M, Hilfiker-Kleiner D, Brunner C, Dübendorfer A, Nöthiger R and Bopp D (1998). Sex-lethal, the master sexdetermining gene in Drosophila, is not sex-specifically regulated in Musca domestica. Development 125: 1487-1494.

Nunney L (1983). Sex differences in larval competition in Drosophila melanogaster: The testing of a competition model and its relevance to frequency dependent selection. Am Nat 121: 67-93.

Nur U (1989). Reproductive biology and genetics. Chromosomes, sex ratios and sex determination. In: Rosen D (ed) Armoured Scale Insects, Their Biology, Natural Enemies and Control. Vol. A. Elsevier Science Publishers b.v.: Amsterdam, pp $179-190$.

Oddie KR (2000). Size matters: competition between male and female great tit offspring. J Anim Ecol 69: 903-912.

Raymond CS, Shamu CS, Shen MM, Selfert KJ, Hirsch B, Hodgkin J, Zarkower D (1998). Evidence for evolutionary conservation of sex-determining genes. Nature 391: 691-695.

Rice WR (1987). The accumulation of sexually antagonistic genes as a selective agent promoting the evolution of reduced recombination between primitive sex-chromosomes. Evolution 41: 911-914.

Rice WR (1994). Degeneration of a nonrecombining chromosome. Science 263: 230-232.

Rigaud T, Juchault P, Mocquard JP (1997). The evolution of sex determination in isopod crustaceans. Bioessays 19: 409-416.

Rocha LS, Perondini ALP (2000). Analysis of the sex ratio in Bradysia matrogrossensis (Diptera, Sciaridae). Genet Mol Biol 23: 97-103.

Schmidt R, Hediger M, Nothinger R, Dubendorfer A (1997). The mutation masculinizer (man) defines a sex-determining gene with maternal and zygotic functions in Musca domestica L. Genetics 145: 173-183.

Sheldon BC (1998). Recent studies of avian sex ratios. Heredity 80: $397-402$.

Sievert V, Kuhn S, Traut W (1997). Expression of the sex determining cascade genes Sex-lethal and doublesex in the phorid fly Megaselia scalaris. Genome 40: 211-214.

Stamps JA (1990). When should avian parents differentially provision sons and daughters? Am Nat 135: 671-685.

Steinemann-Zwicky M, Amrein H, Nöthiger R (1990). Genetic control of sex determination in Drosophila. Adv Genet 27: 189-237. 
Taylor PD (1994). Inclusive fitness arguments in genetic models of behaviour. J Math Biol 34: 654-674.

Taylor PD, Frank SA (1996). How to make a kin selection model. J Theor Biol 180: 27-37.

Trivers RL (1974). Parent-offspring conflict. Am Zool 14: 249-264.

Trivers RL, Willard DE (1973). Natural selection of parental ability to vary the sex ratio of offspring. Science 179: 90-92.

Ullerich FH (1984). Analysis of sex determination in the monogenic blowfly Chrysoma rufifacies by pole cell transplantation. Mol Gen Genet 193: 479-487.

van Hoof JARAM (1997). The socio-ecology of sex ratio variation in primates: evolutionary deduction and empirical evidence. Appl Anim Behav Sci 51: 293-306.

Viets BE, Ewert MA, Talent LG, Nelson LG, Nelson CE (1994). Sex determining mechanisms in squamate reptiles. J Exp Zool 270: $45-56$.

Werren JH (1987). The coevolution of autosomal and cytoplasmic sex ratio factors. J Theor Biol 124: 313-334.

Werren JH, Beukeboom LW (1998). Sex determination, sex ratios and genetic conflict. Ann Rev Ecol Systemat 29: 233-261.

Werren JH, Hatcher MJ (2000). Maternal-zygotic gene conflict over sex determination: effects of inbreeding. Genetics 155: 1469-1479.

Werren JH, Nur U, Wu CI (1988). Selfish genetic elements. Trends Ecol Evol 3: 297-302.

White MJD (1973). Animal Cytology and Evolution. Cambridge University Press: Cambridge.

Wilkins AS (1995). Moving up the hierarchy: a hypothesis on the evolution of a genetic sex determination pathway. BioEssays 17: 71-77. 